\title{
Numerical Analysis of Digital Twin System Modeling Methods Aided by Graph-Theoretic Combinatorial Optimization
}

\author{
Sujing Zhou \\ Henan Railway Traffic Intelligent Safety Engineering Technology Research Center, \\ Zhengzhou Railway Vocational and Technical College, Zhengzhou, Henan 451460, China
}

Correspondence should be addressed to Sujing Zhou; 10516@zzrvtc.edu.cn

Received 17 November 2021; Revised 15 December 2021; Accepted 27 December 2021; Published 2 February 2022

Academic Editor: Gengxin Sun

Copyright $(92022$ Sujing Zhou. This is an open access article distributed under the Creative Commons Attribution License, which permits unrestricted use, distribution, and reproduction in any medium, provided the original work is properly cited.

This paper combines the digital twin system modeling method to conduct an in-depth study and analysis of graph-theoretic combinatorial optimization. This paper provides new ideas and approaches for optimal numerical analysis work by studying the digital twin modeling method that integrates digital modeling and graph theory combination, provides theoretical support for safe, stable, and economic operation of the system, proposes a solution for digital twin model based on big data platform, focuses on the nearest neighbor propagation (AP) and graph theory combination, solves the digital twin real-time monitoring data asynchronous, incomplete problem, and applies the algorithm to the digital twin model based on the big data platform for data preprocessing to achieve better results. This paper also presents a web-based digital twin system based on intelligent practical needs, analysis, and comparison of existing models, combined with digital twin technology, detailing the differences and connections between the various levels of numerical analysis and the implementation of this data in various fields, such as user management, equipment health management, product quality management, and workshop 3D navigation and detailed modeling of the digital twin system based on this numerical analysis to realize remote online monitoring, analysis, and management. In this paper, for the numerical analysis process, firstly, the key technologies of modeling and simulation operation control of production line based on digital twin are studied, and the rapid response manufacturing system based on a digital twin is designed and validated. Secondly, a scheduling technology framework for capacity simulation evaluation and optimization is established, and batching optimization, outsourcing decision, and rolling scheduling techniques are thus proposed to form a batching optimization algorithm based on priority rules, which realizes batching processing, outsourcing decision, and rolling scheduling of production orders to optimize equipment utilization and capacity. Finally, digital twin-based modeling is designed, and the validation results demonstrate the system's superior performance in achieving information interaction between physical and virtual production lines, optimization of numerical analysis, and display of results.

\section{Introduction}

With the continuous development and progress of the manufacturing industry and information technology, the manufacturing industry is facing the great impact and challenges brought by the information age, and the crossfertilization of the manufacturing industry and information technology is also inevitable. Manufacturing is one of the core industries of the country and a huge guarantee to accelerate development and narrow the gap with developed countries. Therefore, it is urgent to improve the level of the manufacturing industry. Along with the rapid economic growth and the continuous improvement of material level, the labor cost has also risen, and most of the traditional manufacturing industries that rely on cheap labor to develop have been on the verge of elimination. However, in the modern manufacturing industry, intelligent equipment represented by industrial robots and automatic transportation devices have brought a huge impact and industrial changes to the traditional manufacturing and coordination delivery and other related industries' production and distribution methods. The process of informatization and digitization of the manufacturing industry is particularly important [1]. In the transformation from traditional mechanical production to intelligent and automated modern industrial production, informatization and digitization are 
essential parts. Production line, as the core component in industrial automation, is a very common machine system used in modern industry [2]. Therefore, modeling simulation, operation control, and capacity evaluation of production lines are particularly important.

Advances in science and technology and changes in market demand are the drivers of industrial change and the catalysts for production models. Digital twin is the use of physical equipment, digital models, historical data, information interaction, and other data to integrate multidimensional, multidisciplinary, and multifunctional simulation process and to complete the mapping of reality in the digital space and the process of representing the life cycle of physical equipment. It is a concept beyond reality which can be seen as a digital mapping system of several important and related physical devices. Following the third industrial revolution, the market demand, which is mainly characterized by multi-variety and small-lot production, requires higher flexibility in enterprise production systems. As a flexible production organization mode, the unitized production model can not only eliminate the rigid defects of traditional fixed assembly lines but also improve the efficiency and response speed of production systems. Based on the study and research related to digital twin and data analysis, the data preprocessing-related technology for digital twin model is studied in-depth, and the construction scheme of digital twin model for graph theory combination based on big data platform is proposed. At the same time, data preprocessing in the digital twin model is realized using the existing data, which improves efficiency [3]. A digital twin is a process of using physical devices, digital models, historical data, information interaction, and other data to integrate a multi-dimensional, multi-disciplinary, and multi-functional simulation process to complete the mapping of reality in digital space and represent the life cycle of physical devices; it is a concept beyond reality and can be regarded as a digital mapping system of several important and related physical devices [4]. The theoretical and technical system of the digital twin is universally adaptable to human life and production and has many suitable application scenarios, such as industrial product design and manufacturing, innovative medical analysis, engineering project construction, and other scenarios. The application of engineering project construction is by far the most in-depth, while intelligent manufacturing is the one with the most attention and the hottest research.

Machine tools are the key equipment in the processing and manufacturing process, and with the development of science and technology, machine tools are constantly updated and iterated. Based on the discussion and application of the digital twin model by the industry and scholars, we find that the digital twin model is a kind of information mirroring model, virtual and real interconnection model, which exists in the whole life cycle of physical objects and coevolves with it, and how to ensure data transmission in real time and accuracy is particularly important. With the policy support of the manufacturing industry, numerical control technology has been vigorously promoted. In terms of ordinary machine tools, CNC machine tools have a high output ratio, high degree of digitalization, reliable processing results, stable processing level, etc. In the early stage of the machine tool processing program design and production preparation, the $\mathrm{CNC}$ machine debugging cycle is a long, risky, and high investment [5]. At the same time, the CNC machine tool technicians also have a high level of technical requirements. Therefore, in the actual production process, the CNC machine tool machining process design errors will bring serious processing accidents. The digital twin-based production line modeling and simulation operation control technology aims to realize the real-time interaction of data information between the production line in physical space and its corresponding information space, to simulate the modeling of the production line and evaluate and optimize the data analysis, and to improve the efficiency and effectiveness of enterprises and enhance the independent research and development capability and scientific and technological innovation capability of the national defense science and technology industry.

\section{Related Works}

Advances in science and technology and changes in market demand are the drivers of industrial change and the catalysts for production models [7]. With the rapid growth of our country's economy and the continuous improvement of material levels, labor costs have also risen, and most of the traditional manufacturing industries that rely on cheap labor for development have been on the verge of being eliminated. However, in the modern manufacturing industry, intelligent equipment represented by industrial robots and automatic transportation devices have brought huge impacts and industrial changes to the production and distribution methods of traditional manufacturing and logistics transportation and other related industries. The process of informatization and digitization of the manufacturing industry is particularly important. Following the third industrial revolution, the market demand, which is mainly characterized by multivariety and small-lot production, requires higher flexibility in enterprise production systems. As a flexible production organization mode, the unitized production model can not only eliminate the rigid defects of traditional fixed assembly lines but also improve the efficiency and response speed of production systems. Based on the study and research related to digital twin and data analysis, the data pre-processing related technology for digital twin model is studied in-depth, and the construction scheme of digital twin model for graph theory combination based on big data platform is proposed [8]. At the same time, data pre-processing in the digital twin model is realized using the existing data, which improves efficiency [9]. The digital twin is a process of using physical devices, digital models, historical data, information interaction, and other data to integrate a multi-dimensional, multi-disciplinary and multi-functional simulation process to complete the mapping of reality in digital space and represent the life cycle of physical devices; it is a concept beyond reality and can be regarded as a digital mapping 
system of several important and related physical devices. The theoretical and technical system of the digital twin is universally adaptable to human life and production and has many suitable application scenarios, such as industrial product design and manufacturing, innovative medical analysis, engineering project construction, and other scenarios [10]. The application of engineering project construction is by far the most in-depth, while intelligent manufacturing is the one with the most attention and the hottest research.

Machine tools are the key equipment in the processing and manufacturing process, and with the development of science and technology, machine tools are constantly updated and iterated. Relevant companies have also begun to introduce unitized production with this wave. Combined with the current production process of the company, they have implemented a unit production model for existing production equipment, manufacturing processes, and operators, thereby improving the company's production efficiency and increasing production. The line is flexible, guarantees product quality, and shortens product delivery time. With the policy support of the manufacturing industry, numerical control technology has been vigorously promoted [11]. In terms of ordinary machine tools, CNC machine tools have a high output ratio, high degree of digitalization, reliable processing results, stable processing level, etc. In the early stage of the machine tool processing program design and production preparation, the $\mathrm{CNC}$ machine debugging cycle is a long, risky, and high investment. At the same time, the CNC machine tool technicians also have a high level of technical requirements [12]. Therefore, in the actual production process, the $\mathrm{CNC}$ machine tool machining process design errors will bring serious processing accidents [13]. The digital twin-based production line modeling and simulation operation control technology aims to realize the realtime interaction of data information between the production line in physical space and its corresponding information space, to simulate the modeling of the production line and evaluate and optimize the data analysis, and to improve the efficiency and effectiveness of enterprises and enhance the independent research and development capability and scientific and technological innovation capability of the national defense science and technology industry.

\section{Digital Twin System Modeling Approach Aided by Graph-Theoretic Combinatorial Optimal Numerical Analysis Construction}

3.1. Digital Twin System Design. Big data provides data computation, data storage, and the ability to share data processing. The digital twin maps the physical model correspondingly into a digital model, and the two can exchange data with each other to continuously optimize and improve the performance of the physical entity by interacting with the data. This section combines big data with digital twin technology and applies it to the operation and maintenance phase of the rolling stock to build a digital twin model of the rolling stock based on the big data platform.
(1) Sense layer: it is also known as the data acquisition layer; the main role is to send the collected sensor's real-time monitoring data to the data layer for transmission.

(2) Physical entity layer: the physical layer contains multiple physical entities, which can be some components, devices, or system-level physical bodies; digital twins create virtual models corresponding to the physical entities, i.e., constructing digital twins, interacting with the physical models for virtual-real interaction, updating feedback, iterative optimization, etc., to provide intelligent and efficient services for each physical entity.

(3) Cloud network layer: the cloud network layer exists with each digital twin corresponding to the physical entity target object in the physical entity layer, which is also a digital model reflecting a certain feature of the physical object and has two main functions: one is modeling management, involving digital modeling and visual display of the physical object, synchronous update, and operation management with the physical object model state; the other is to provide model simulation functions.

(4) Storage device layer: it provides storage areas for the state information that needs to be stored by the respective physical entities and digital twins.

(5) Information communication layer: firstly, the information communication group in the information communication layer consists of several digital twins, and the composition can be decided according to the specific needs, and several digital twins in the group can communicate with each other and exchange information, and secondly, each information communication group can also connect and communicate with each other.

(6) System application layer: its main role is to perform information processing, system management, data processing, data mining, and operation and maintenance analysis.

(7) User layer: this layer is the user domain that uses the digital twin, which contains the human-computer interaction, application software, and shared twin, i.e., other related digital twins. The main purpose is to visualize the information through human-computer interaction and the user can make decisions based on the visualization (Figure 1).

Through the definition of digital twin and the theoretical analysis of the digital twin model, in a certain sense, the application of digital twin technology is the construction of digital twin models of actual physical entities. The thermal system represented by thermal power generating units, with its complex equipment and huge system, is a typical nonlinear, multi-parameter, and strongly coupled complex thermal system. Thermal power plant thermal system modeling methods can be divided into two approaches: mechanism modeling and data-driven modeling. The digital twin stamping production line requires the realization of 
virtual and real mapping between physical space and virtual space. The production line in the physical space is the main body to complete the production activities and has the characteristics of complexity and variety. Among them, mechanistic modeling is to build a model reflecting the intrinsic relationship between the parameters of the unit under the assumption of reasonable simplification and using the law of energy and mass conservation as the theoretical basis [14]. Therefore, mechanism modeling requires a deeper understanding of the modeling object and the determination of a varying number of parameters in the mathematical model based on structural and operational parameters. Therefore, the process of mechanical modeling is more complex. The data-driven machine learning modeling approach is based on statistical principles, ignoring the internal mechanism of the object, estimating the relationship between the input and output based on known data samples, and using computer and artificial intelligence techniques to build the model to predict and judge the unknown output. The fluid network modeling method for thermal systems is a typical mechanism modeling method based on the laws of conservation of mass, momentum, and energy. In the production process of thermal power plants, fluids such as steam, water, oil, and air exist in polymorphic form and are distributed and intersected into a complex fluid network through different pipelines. Because of pipe resistance losses, heat exchange, pump-action, and so on, fluid flow in the thermal network will produce pressure and temperature changes. This change is in compliance with the law of conservation of mass, momentum, and energy abstract the fluid pipeline system as a fluid network. According to the need to calculate the solution, in the fluid network system to select a sufficient number of control bodies, the establishment of mass, momentum energy conservation equations, together with the equipment characteristics equation can calculate the pressure, flow, and temperature distribution of the whole thermal network. Each of the different thermal subsystems can be considered as a system of fluid networks with different topologies. The simulation model of each system consists of a fluid network subsystem module describing the system mass conservation, momentum conservation, and energy conservation relationships and several equipment modules describing the thermodynamic properties of the equipment, where the fluid network system module and the equipment modules are uniquely connected by topological parameters. The high-accuracy fluid network model is important for the correct analysis of the dynamic characteristics of the whole system.

$$
p_{i}-p=\frac{w_{1}-w}{p C_{2}}
$$

Order batching is a nondeterministic polynomial problem. The problem cannot be solved effectively if it is solved according to the traditional exact algorithm. The solutions for order batching are as follows. (1) Priority rule algorithm, which first assigns priority to coordination orders according to the rules and then batches or combines the orders according to the priority. (2) Seeding algorithm, which seeds selection, according to the corresponding rules and then selects other orders corresponding to the seed. (3) Saving algorithm, which compares the sorting distance saved after merging two orders, sorts the saved values, and performs batching based on the sorting. (4) Heuristic algorithm to construct a mathematical model considering the walking distance of workers and use the heuristic algorithm to solve the batching problem. The batching optimization technology-oriented to capacity simulation and evaluation optimization will adopt the algorithm based on priority rules to batch the orders and establish the optimization objectives in five aspects, such as production cycle time, delivery satisfaction, equipment utilization, and production cost of workpiece sub-batch quantity and then consider the process preparation time before order production for the characteristics of efficient intelligent processing production line of aerospace complex compartments [15]. The digital twinoriented production scheduling system is driven by the production operation plan and is the executor of the operation plan. It is mainly used to simulate, evaluate, and optimize the production line capacity, adjust and control the daily production activities of the production line, and adjust the entire control process. It is a process of real-time feedback and dynamic adjustment. According to general processing experience, it is not difficult to conclude that while processing, some equipment resources are not fully mobilized and are in an idle state because the workpiece to be processed in the equipment is still being processed in the previous process, and enterprises are often processed after the first process of an order and then the second process; it can be neat for statistical purposes, but it reduces the utilization rate of the equipment and even leads to orders not be timely. The order is not delivered in time. If the order is divided into batches and the second process is carried out after the first process of a batch of orders, not only can the equipment utilization rate be improved but also the production capacity can be increased. However, it is not better to divide the order into smaller batches because factors such as workpiece handling time must also be considered, which is shown in Figure 2.

The digital twin stamping production line requires to realize the mapping of physical space and virtual space. The production line in the physical space is the main body to complete the production activities, which has the characteristics of complexity and diversity. In the process of constructing the physical data model of the production line, it is necessary to define and classify and analyze the manufacturing resources and production activities within the scope of the production line and establish the physical data model. In the process of building the digital information model of the production line, it is also necessary to formally describe the manufacturing resources and manufacturing capabilities of the production line, express them clearly, and abstract them into corresponding knowledge descriptions so that they can be recognized by the computer to realize various operations on manufacturing resources, which is an indispensable key to establish the digital twin stamping production line model. 


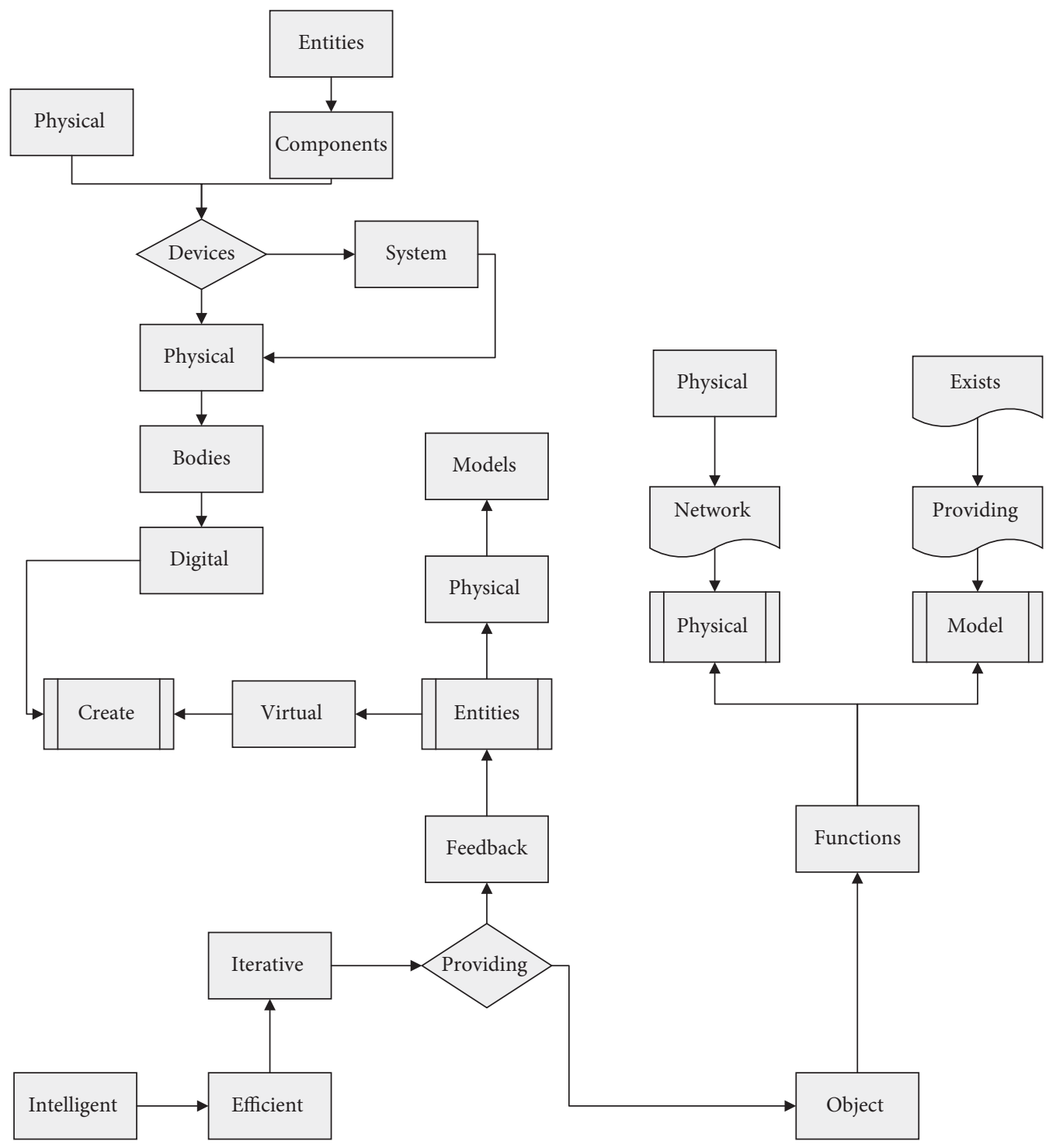

Figure 1: Data-driven modeling flowchart.

$$
\lambda=\left(\frac{K_{1}}{D}\right)^{2} .
$$

To ensure the calculation accuracy of the model, from the principle of meeting the application needs and reflecting the advanced system, the established thermal system model should have the characteristics of consistency, intelligence, and real time. The fluid network mechanism simulation model adopts the idea of modular modeling, and the module division scheme usually takes the independent thermal equipment as the basic module, which can mathematically ensure the independence and compatibility between modules.

3.2. Graph-Theoretic Combinatorial Optimality Analysis. Heterogeneous element fusion of manufacturing service energy consumption process in physical space refers to the identification and sense of heterogeneous information of people, machines, objects, environment, and space involved in manufacturing service manufacturing in physical space by sensing devices, transmission to data support layer by the network, integration of multisource heterogeneous fusion data through correlated data of energy consumption information, and real-time feedback interaction between physical space and information space, which makes physical space people, machines, objects, environment, space, energy consumption elements, and other information with the virtual space of them highly collaborative. The fusion of heterogeneous elements of physical space manufacturing service energy consumption assessment process is to realize the fusion of multiple models of virtual space manufacturing service energy consumption assessment and multiple elements of energy consumption assessment, as shown in Figure 3.

The performance of manufacturing service energy consumption assessment in virtual space is as follows: based on the manufacturing service process information in physical space, design models, process models, 


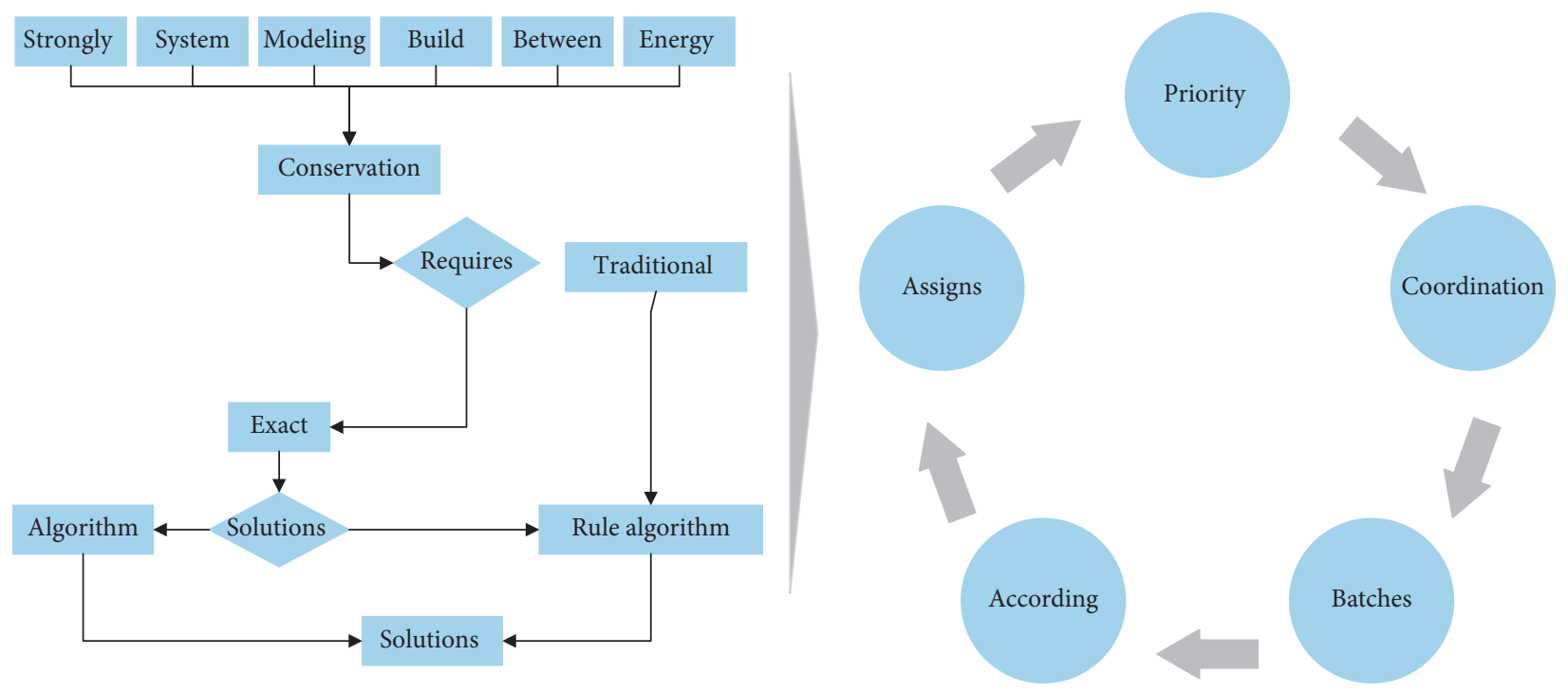

Figure 2: Model fusion diagram.

manufacturing models, assembly models, usage models, logistics models, forward and reverse supply chain models, recycling models, market analysis models, and so on are created in virtual space corresponding to them, and they can reflect the scenes, environment, materials, and equipment and energy consumption levels in physical space. The models reflect the data and simulation information of different manufacturing service stages, among which the product design model reflects the information of user and market demand for the designed product, the rough geometry size information of the product, the manufacturing plant information, the product manufacturing process flow information, the recycling plan information, and so on; the process model reflects the process optimization information of a certain manufacturing stage of the product, the information of different load adjustment relationship, the material, and equipment. The process model reflects the process optimization information, different load adjustment relationship information, material and equipment rational arrangement information, layout optimization information, scene information, and so on; the manufacturing model reflects the geometric dimension information, product behavior information, object feature information, physical property information, parts information, and so on of the accurate 2D-3D model of the product; the assembly model reflects the child-parent relationship information, installation sequence and step information, assembly method and debugging scheme information, and plane and 3D assembly dimension chain information. The usage model reflects the information of product service life and remaining life, basic information of users, operation information, basic information of products, failure information of products in use, and so on; the recycling model reflects the information of reasons and extent of product. In addition, other models in virtual space also reflect information on material properties, processability of materials and parts, material damping, environmental pollution, waste emission, forecast and simulation, equipment, physical operation parameters, process parameters, consumption of different energy sources, human resources, space consumption, time loss, lack of production capacity, time loss information, production capacity shortage information, and high energy consumption link information. Through the fusion of multiple models, simulation, prediction, evaluation, and analysis, the information of different models is integrated into a complex fusion model that can reflect the whole life cycle information of manufacturing services, i.e., the digital twin model.

$$
\left[\begin{array}{cc}
S_{1} & 0 \\
1 & S_{2}
\end{array}\right] \cdot\left(\begin{array}{l}
x \\
y
\end{array}\right)=\left(\begin{array}{ll}
s_{1} & x \\
s_{2} & y
\end{array}\right) .
$$

The digital twin-oriented production scheduling system is driven by the production operation plan and is the executor of the operation plan, which is mainly used to simulate and evaluate and optimize the production capacity of the production line and to regulate and control the daily production activities of the production line, and the whole regulation and control process is real-time feedback and dynamic adjustment process [16]. On the one hand, the production line needs to receive the part processing tasks issued by the upper-level planning system, such as the number of orders, part types, part processes, process quantities, delivery times, and equipment resource quantities to prepare the production line operation plan, which can allocate the production tasks to each processing equipment concretely and reasonably. As far as ordinary machine tools are concerned, CNC machine tools have the characteristics of high output ratio, high degree of digitization, reliable processing results, and stable processing level. In the processing program design and production preparation of the machine tool, the $\mathrm{CNC}$ machine tool has a long debugging cycle, high risk, and high investment. On the other hand, the production line needs to be balanced according to the actual implementation of the production line and the emergence of abnormal conditions, such as the emergence of various types 


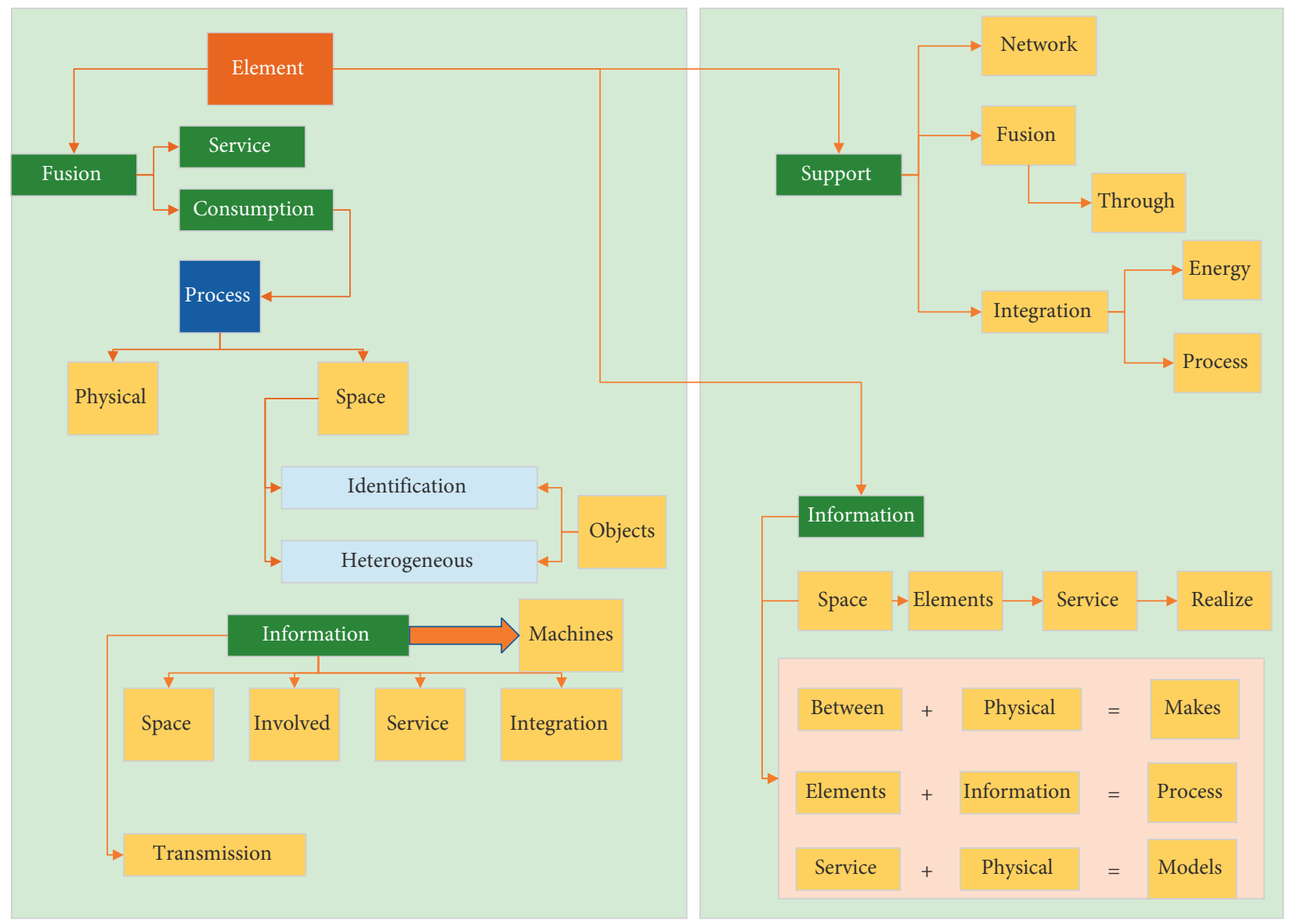

FIgURE 3: Schematic diagram of the graph theory combination.

of disturbance events, when the operation plan arrangement needs to be revised to ensure the processing progress, and if necessary, rescheduling can be carried out, and then the production line operation can be scheduled effectively. In this paper, a two-level progressive production scheduling strategy is used to make the scheduling results conform to the actual production activities of the production line. Production line production scheduling mainly includes two aspects: the job sequencing layer and the job control layer, i.e., static scheduling and dynamic scheduling of jobs, which are complementary to each other (Figure 4).

To meet the requirements of energy saving and emission reduction in the manufacturing industry, energy consumption is predicted and assessed at the process level, equipment level, product level, and workshop level. Production and manufacturing system is the research target from the whole life cycle of product design, manufacturing, assembly, use, recycling, and transportation. The focus of energy consumption assessment differs from the target of different assessments. The digital twin conceptual model includes three parts: physical products in the real space, virtual products in the virtual space, and the data information chain connecting the physical information space. Discrete and continuous production methods: different from the discrete manufacturing services, continuous manufacturing services such as steel and chemical have a large energy consumption of single machine electromechanical equipment and instruments. Whether more energy-efficient electromechanical equipment or instruments can be designed will directly affect the energy consumption or energy efficiency of manufacturing services. The process of discrete manufacturing services is complex, and the manufacturing service processes are interrelated, so its research focuses more on each link, including modeling, analysis, and prediction of energy consumption under different equipment, different process routes, and different production environments. Large-volume discrete manufacturing services, on the other hand, are studied to reduce the energy consumption per unit of a product using energy consumption assessment of mass production. Product manufacturing and service levels: product manufacturing often focuses on the assessment of the energy consumption status of various types of equipment involved in the each stage of production process and focuses on the reduction of the overall energy consumption of the product manufacturing system through prediction and simulation. The energy consumption assessment of manufacturing services, on the other hand, is a study based on manufacturing products and aims to promote users' access to high-quality and low-consumption manufacturing services. This energy efficiency assessment is an extension of product energy consumption assessment, which combines 


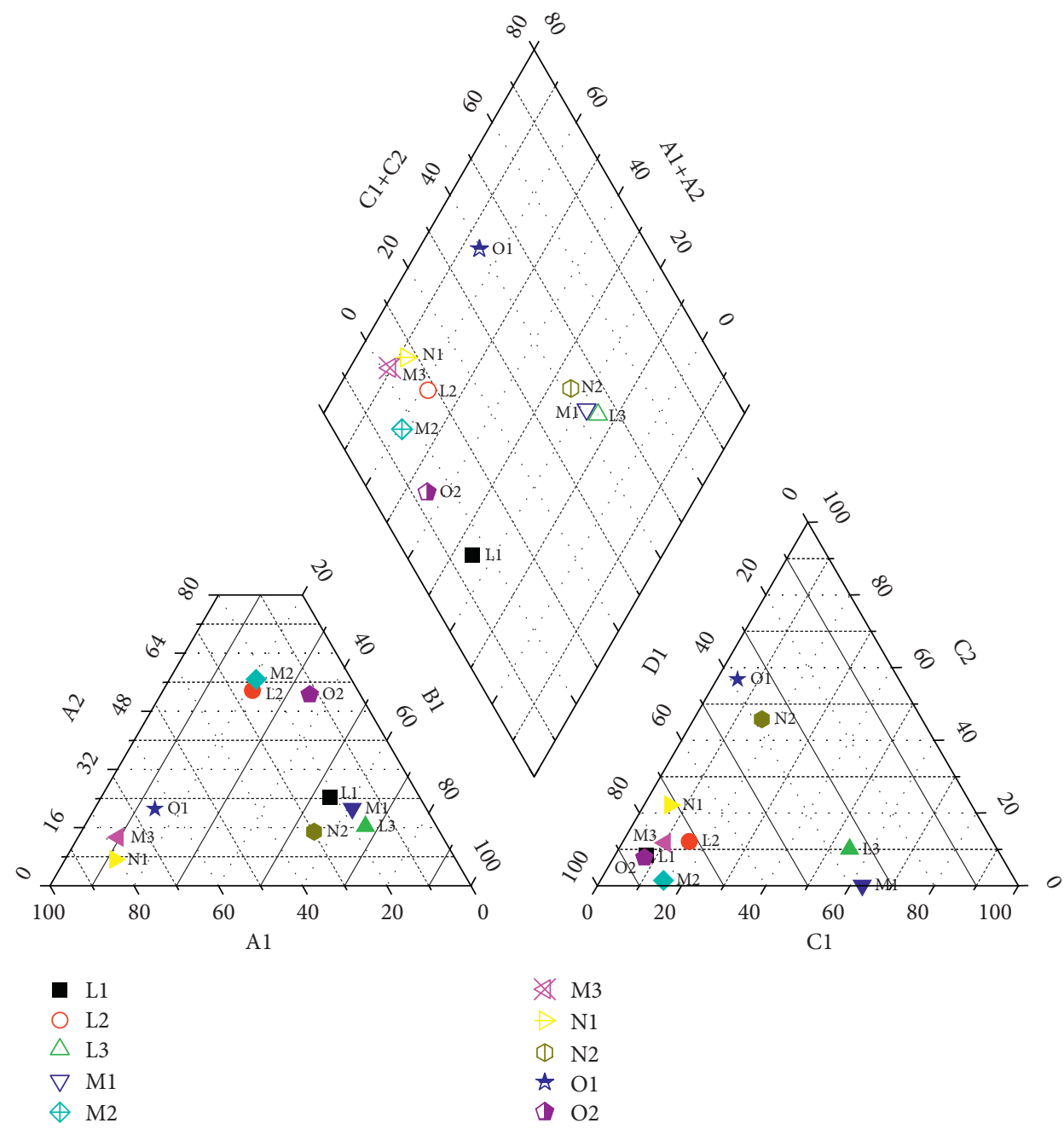

Figure 4: Conversion schematic.

theories and methods oriented to manufacturing services and assesses them from the dual perspectives of energy consumption and service quality.

$$
E P=\{\phi \cdot I S \cdot \operatorname{Em}(r)\} .
$$

Based on the established offline intelligent recognition mechanism twin model, the online collaborative updating of the digital twin model parameters and the real-time correction of the offline model parameters are realized according to the cooperation and fusion theory of the digital twin model. The parameter curves under full working conditions are then refitted to ensure the tracking capability of the model for the actual system for subsequent test prediction. To further improve the accuracy and robustness of the model, a multi-model output online fusion strategy using moving pane information entropy is used to achieve online fusion of the digital twin model with the established improved instantaneous learning strategy data-driven model. The growth in the number of data objects leads to the simultaneous growth in the processing time of the algorithms running on both single machines and clusters. With the increase in product types, the continuous shortening of product life cycles, and increasingly personalized customer needs, traditional mass production models cannot cope with such development changes. Under the situation that the production system needs to meet today's multi-variety, small-batch, and higher flexibility requirements, people began to try to use a new production mode-unit production mode, and this production mode not only meets the growing individual needs of customers but also the resulting problem of rigidity of the production system is reduced. When the algorithm is run serially on a single machine, an exponential form of growth in the preprocessing time of the entire motley monitoring data occurs. However, the increase in the running time of the algorithm on the cluster is significantly lower than that of the single machine. This indirectly proves that the algorithm is more efficient in processing the rolling stock monitoring data relative to running in the normal single-machine mode.

The digital twin conceptual model contains three parts: the physical product in real space, the virtual product in virtual space, and the data information chain connecting the physical information space. In the virtual space, it is possible not only to visualize the desired lightweight model of the product and its associated properties but also to verify performance simulations in advance, including 
physical behavior and real-time and maximum computational load. The data-driven machine learning modeling method is based on statistical principles, ignoring the internal mechanism of the object, estimating the relationship between input and output based on known data samples, and using computer and artificial intelligence technology to build models, so as to understand the unknown output which is predicted and judged. Lightweight models in virtual space mean less wasted communication time and enhanced understanding of deeper levels of product information. A more comprehensive collection of physical entity data information in physical space is needed to better support twin model operations. The data information chain contains data on the physical model, sensor updates, and operational history and is the bridge between the virtual and physical space interconnections [17]. A digital twin is an effective method to achieve real-time interaction and integration between the physical world and the information world, and the study of the connotation of the digital twin helps to deepen the understanding of its application, and it helps to start the research of digital twin production line modeling method. The digital twin is applied to the whole product lifecycle, and the digital twin is built based on fullfactor, full-lifecycle data, where the product lifecycle is defined as seven stages: requirement analysis, conceptual design, detailed design, manufacturing, sales, after-sales service, and recycling. The digital twin concept helps companies establish a bidirectional data flow between all aspects of the full lifecycle, providing full, multi-dimensional, and maximized value to producers and users.

\section{Analysis of Results}

4.1. Digital Twin System Performance Testing. This test is a performance test, using the testing tool to complete the recording of performance data during the operation of the system and using the values of CPU usage, memory usage, and other parameters recorded during the experiment as the benchmark for assessment. The performance test time is one hour. The evaluation criteria are as follows: CPU usage needs to be less than $40 \%$ of the actual CPU total, and memory usage needs to be less than $30 \%$ of the actual memory total. In this experiment, the system is monitored using the resource monitor in the task manager, and the overall performance test results of the system are monitored and recorded for the CPU usage data of key nodes during the performance test, such as the moment of the system startup, the moment of entering the machine configuration page, the moment of machine configuration completion, the moment of contact between the workpiece model and the tool model, the moment of workpiece model machining completion, etc., and the results are plotted. The results of the logging are plotted, which are shown in Figure 5.

Model predictive control (MPC) algorithm is an advanced control technique based on display predictive models that use model multi-step prediction, rolling optimization, and feedback correction strategies to predict and optimize the future dynamic response output of an object. The predictive model is an important part of predictive control, and its main function is to predict the future state or output of the controlled object based on its historical information and future inputs, especially for nonlinear predictive control systems. Predictive control has no strict mandatory requirements on the structural form of the predictive model, but its control effect largely depends on the performance of the predictive model [18]. Obtaining a predictive model that accurately reflects the future changes in the dynamic characteristics of the system is a prerequisite for achieving optimal control. Predictive control is an optimization-based control algorithm that uses a certain performance index to evaluate the control effect of the controlled object. The optimization in predictive control is a kind of finite time rolling optimization, and the optimization process is not carried out offline once, but repeatedly online. Compared with traditional optimal control, predictive control can obtain more accurate control quantity in real time, which is the significance of rolling optimization and the fundamental point that distinguishes predictive control from traditional optimal control. Predictive control is a closed-loop control algorithm. In the operation of complex thermal systems, there are uncertainties in the actual system such as model mismatch or environmental disturbances that cause the predicted output to deviate from the ideal state. Predictive control only achieves the control role at this moment, and by the next sampling moment, the actual output of the object is first monitored and this real-time information is used to make feedback corrections to the model-based prediction before a new optimization is performed. The overall system architecture, system development, and operating environment and key technologies of system development are elaborated. The corresponding database tables are designed according to the functional requirements, and the development of functional modules of the industrial robot reconfigurable digital twin manufacturing prototype system is completed. As can be seen, the optimization in predictive control not only is based on the prediction model but also makes use of the feedback information, which constitutes a closed-loop optimization. Thus, the predictive control algorithm has the advantages of good control, robustness, and model adaptability and is particularly suitable for complex thermal system control processes with nonlinearity, time variation, and large delays.

$$
P D_{s}=\left(B R_{a}, C U, E S R_{S N}\right) \text {. }
$$

The module includes three submodules: experimental design, online monitoring, and real-time control. According to the forming process planned in the above module and by applying the technologies of online monitoring of forming status, multisource information fusion, data processing, intelligent decision making and optimization, the predicted and optimized process parameters and strategies, the SLM forming experiment, and online monitoring and optimization control are carried out. The goal of optimization is to achieve a better quality of formed parts, to optimize the process parameters of the forming process based on the comparison and analysis of prediction data and experimental data, and to adjust the process parameters and strategies of the forming process in real time to compensate 

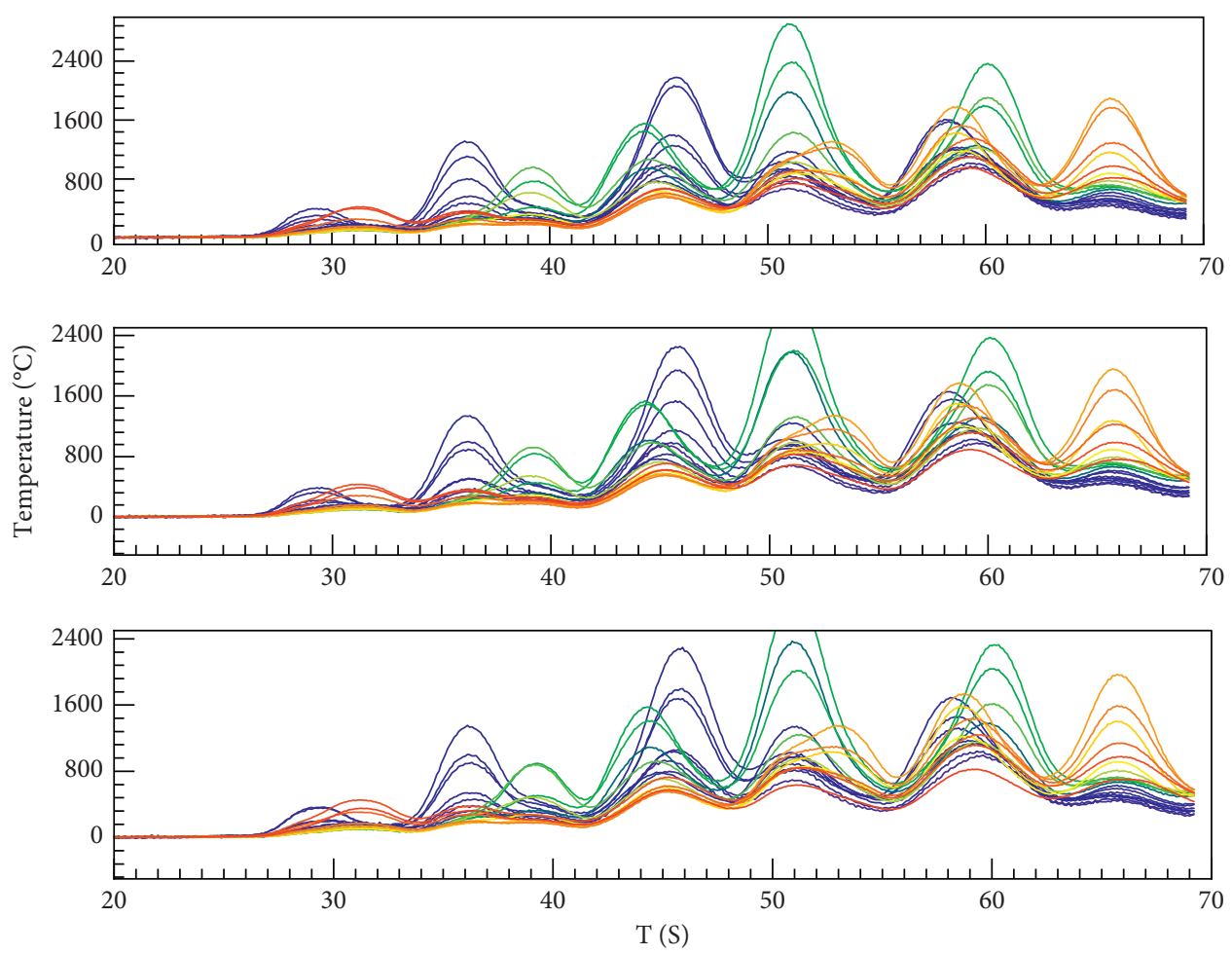

Figure 5: Performance test data graph.

for the defects of the forming process and control the quality of formed parts. Cutting simulation is the core function of this system, and it is also the focus of this test. The CNC machine model used in this test is CK0632, the length of the workpiece to be machined is $100 \mathrm{~mm}$, the width is $100 \mathrm{~mm}$, and a tool head model is a spherical tool (Figure 6). On the basis of the established offline intelligent identification mechanism twin model, according to the coordination and fusion theory of the digital twin model, the online collaborative update of the digital twin model parameters is realized, and the offline model parameters are corrected in real time. The cutting simulation function test is executed several times, a single machining process lasts two minutes, and the machining content is to carry out free-form cutting by the workpiece machining process.

Load-side heating load prediction is the basis for heating to be intelligent and the boundary for calculations performed by the twin model system and also the basis for the optimization of the entire electric boiler heating and heat storage system. The load-side heating load is influenced by a variety of factors, including meteorological factors, temporal factors, social factors, and factors related to the system itself. Based on the real-time tracking capability of the digital twin model, a set of dynamic optimization strategies for intelligent operating conditions of the thermal system based on load distribution and optimization of operating conditions is proposed. Meteorological factors include ambient temperature, wind level, solar radiation intensity, and so on; social factors involve the influence of local population distribution and economic development on the heating system; temporal factors include the factors affecting the heat load of users on working days and holidays. In this paper, the study of load- side heating load forecasting is to realize the load demand situation of each heating unit in the next 24 hours of the day. An adaptive data-driven modeling approach based on meteorological factor clustering and improved on-the-fly learning strategy is applied to heat load forecasting to provide computational bounds for boiler electric load allocation on the supply side. The heating system is a slow time-varying process system, which does not require high real-time performance for the established source-side and load-side local prediction models, and the local modeling approach based on support vector machines and multimodel parameter identification can improve the modeling accuracy through the optimization-seeking process and meet the requirements of online computation for twin model systems. The load-side and source-side load prediction algorithms of the heating system can tap into the energy consumption habits of users, which will provide references for the next stage of optimal load allocation and parameter search algorithms of the heating system.

$$
H V=\frac{P}{W}=1.1 \frac{P}{D}
$$

4.2. Graph-Theoretic Combinatorial Optimality Results. Based on the real-time tracking capability of the digital twin model, a set of intelligent working condition dynamic optimization strategies for the thermal system based on load allocation and working condition seeking is proposed. Taking the electric boiler storage heat supply system as the research object, the digital twin model system is used to predict and calculate the grid load, electric boiler system, and 

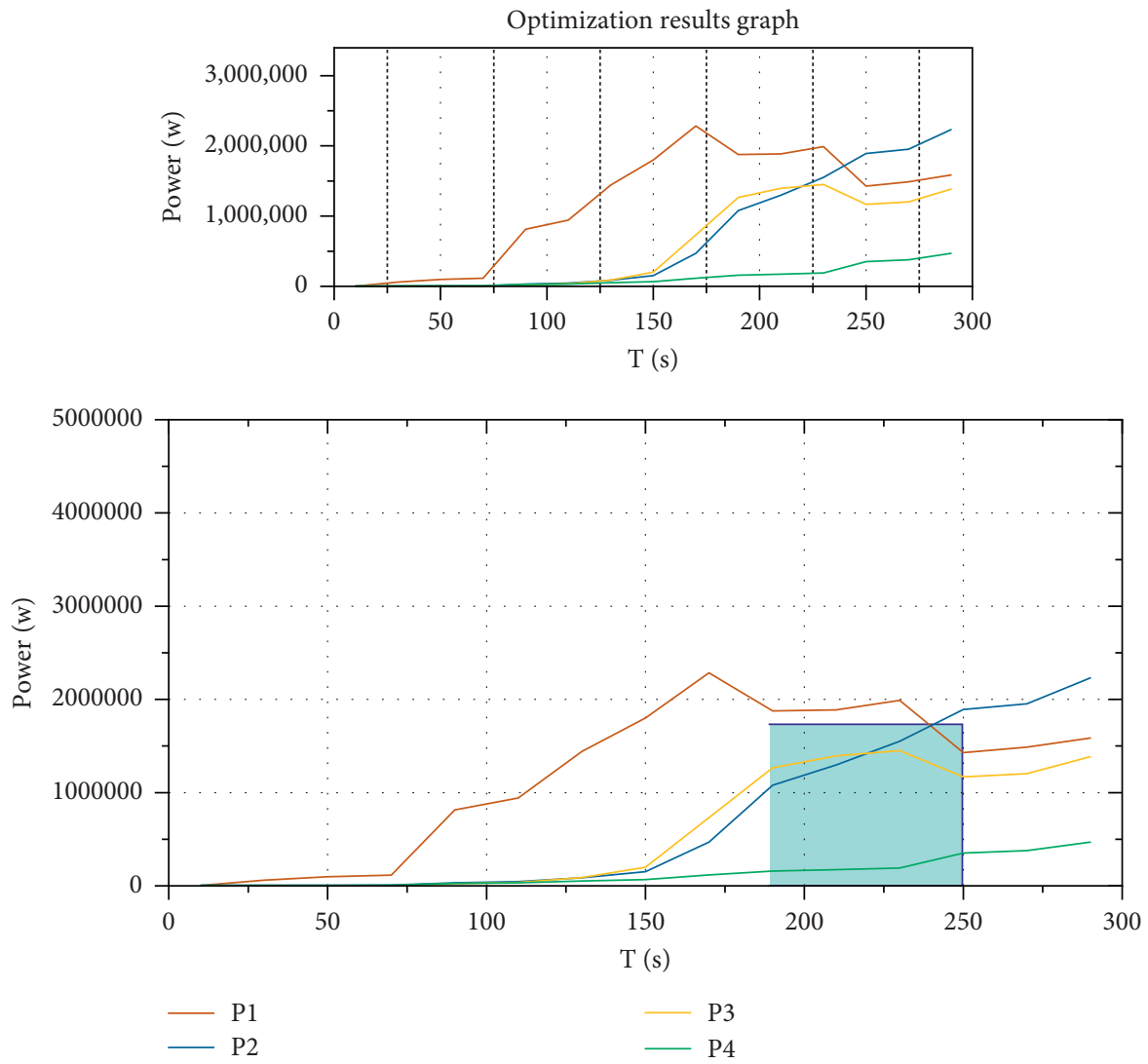

Figure 6: Data monitoring chart.

heat storage system according to the energy cost analysis and load allocation strategy [19]. The dynamic operation of the system under different operation scenarios and working conditions is simulated, and the optimal heating regulation and load distribution strategy is derived. The economic and safe operation of the heat supply and storage system is ensured, and the stable operation of the grid can also be guaranteed. The optimization goal is to achieve better quality of formed parts. According to the comparison and analysis of predicted data and experimental data, single-objective or multi-objective optimization of the process parameters of the forming process is carried out, and the process parameters and strategies of the forming process are adjusted in real time to compensate the forming process. The quality of formed parts is controlled. Cutting simulation is the core function of this system, and it is also the focus of this test. This section also takes the SCR denitrification system of thermal power plants as an example and applies the established adaptive and self-evolving intelligent SCR denitrification system digital twin model to the model predictive control algorithm to control and optimize the system inlet ammonia injection volume, as shown in Figure 7.

The test results show that the adaptive predictive control using the digital twin model is more accurate than the traditional PID control results, which can realize the accurate control of ammonia injection volume and more stable system operation. The reconfigurable digital twin manufacturing prototype system for industrial robots was designed and implemented, detailing the overall system architecture, system development, and operating environment and key technologies for system development, designing corresponding database tables for functional requirements, and completing the development of functional modules for the reconfigurable digital twin manufacturing prototype system for industrial robots. The four functional modules of this system include the virtual twin control module, data interaction and model update module, system reconfiguration module, and humancomputer interaction interface module, which meet the normal operation requirements of the digital twin manufacturing system and improve the flexibility of the digital twin manufacturing system. In order to ensure the calculation accuracy of the model, starting from the principle of meeting application needs and reflecting the advanced nature of the system, the established thermal system model should have the characteristics of consistency, intelligence, and real time. The overall method design all considers the use of digital twin technology to integrate data throughout the parameter optimization system to achieve real-time data construction, transfer, storage, acquisition, decision making, analysis, and feedback and realization. The parameter optimization system improves the intelligence and forming quality of selective laser fusion forming. 


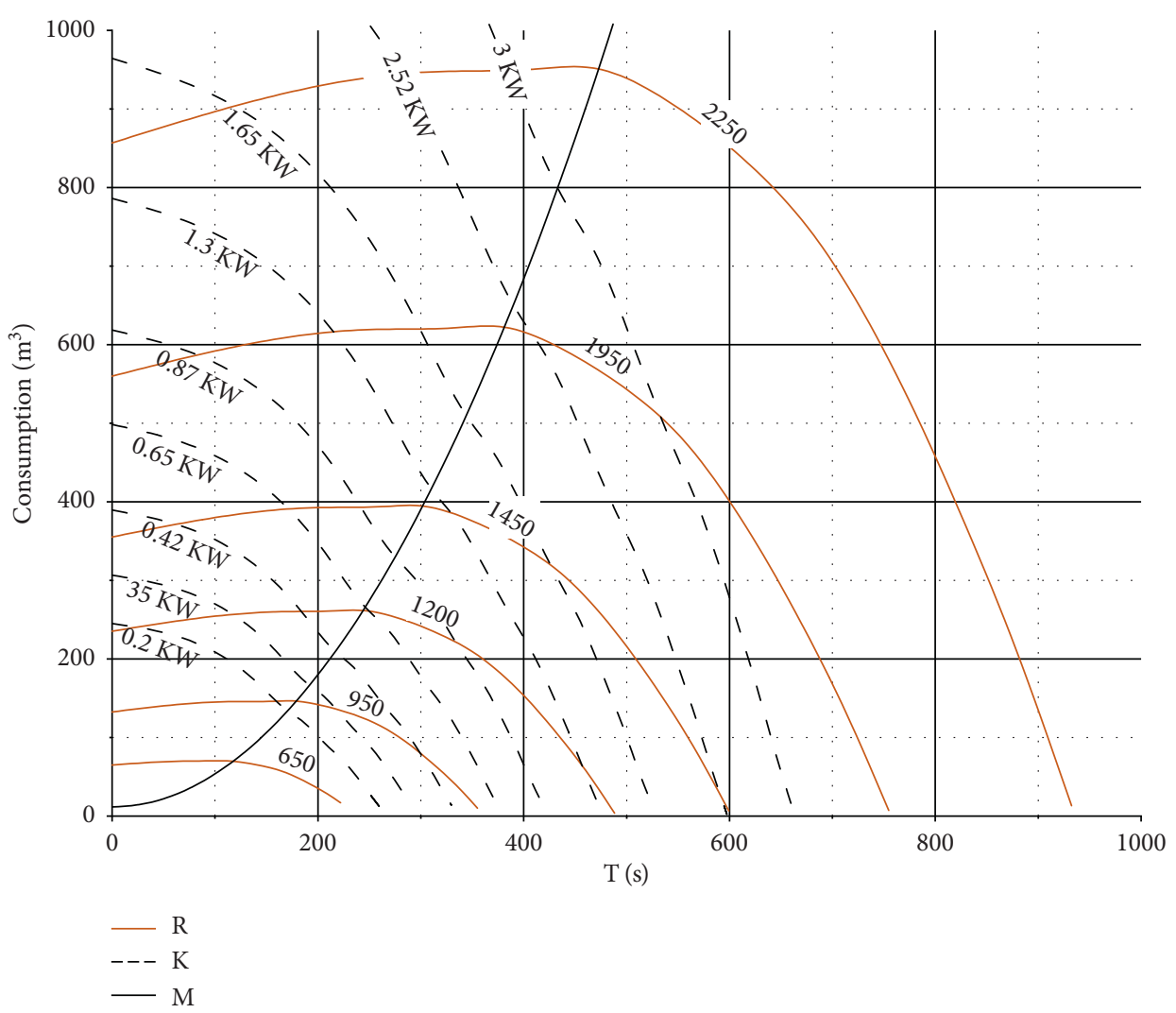

FIGURE 7: Optimization result graph.

$$
k\left(x_{1}, y_{1}\right)=\frac{x_{1}+y_{1}}{y^{2}}
$$

This section gives the design, development, and implementation process of the digital twin knowledge information service system of the stamping production line. The structural framework of the system and the system application platform are built, the database is designed for the functional requirements of the system, and each functional module of the system is analyzed, including the basic information module, the knowledge information module, the task information module, and the twin digital model display module. Finally, the implementation results of each functional module are given, and the system is tested, and the system can operate well. The modeling method provides a new idea for the implementation of a digital twin stamping production line.

\section{Conclusion}

In the context of facing the transformation and upgrading of the manufacturing industry and shifting to smart manufacturing, digital twin, as a new technical means to realize smart manufacturing, has attracted many scholars' research and discussion. Although this concept has been proposed for some time, there are few project applications of a digital twin as a technical tool, and the referenceable experience is almost zero, and the reference content is mostly theoretical and conceptual research. In this paper, we combine the digital twin system modeling method with indepth research and analysis of graph-theoretic combinatorial optimization and the design, debugging, and verification of the digital twin-based rapid response manufacturing system. The upgrade of the system is completed, and the digital twin functions such as user management, equipment health management, product quality management, and workshop 3D navigation are realized, which provide support for enterprises to implement intelligent manufacturing. Along with the development of big data technology, sensing technology, and IoT technology, modeling technology begins to integrate with big data, IoT, artificial intelligence, and other technologies. Data-driven modeling techniques are applied and combined with mechanism modeling to build a high-precision real-time adaptive tracking digital twin model that can simulate and even track and extrapolate the real unit dynamic process. The implementation of advanced control strategies and optimization methods can first be tested, analyzed, and optimized on the digital twin model system.

\section{Data Availability}

The data used to support the findings of this study are available from the corresponding author upon request.

\section{Conflicts of Interest}

The author declares that there are no conflicts of interest. 


\section{References}

[1] S. Mohajer, I. Bergel, and G. Caire, "Cooperative wireless mobile caching: a signal processing perspective," IEEE Signal Processing Magazine, vol. 37, no. 2, pp. 18-38, 2020.

[2] C. Zhuang, J. Liu, H. Xiong, and X. Ding, "Connotation, architecture and trends of product digital twin," Computer Integrated Manufacturing Systems, vol. 23, no. 4, pp. 753-768, 2017.

[3] P. Jain, J. Poon, J. P. Singh, C. Spanos, S. R. Sanders, and S. K. Panda, "A digital twin approach for fault diagnosis in distributed photovoltaic systems," IEEE Transactions on Power Electronics, vol. 35, no. 1, pp. 940-956, 2019.

[4] F. Tao, H. Zhang, A. Liu, and A. Y. C. Nee, "Digital twin in industry: state-of-the-art," IEEE Transactions on Industrial Informatics, vol. 15, no. 4, pp. 2405-2415, 2018.

[5] B. Schleich, N. Anwer, L. Mathieu, and S. Wartzack, "Shaping the digital twin for design and production engineering," CIRP Annals, vol. 66, no. 1, pp. 141-144, 2017.

[6] C. Gehrmann and M. Gunnarsson, "A digital twin based industrial automation and control system security architecture," IEEE Transactions on Industrial Informatics, vol. 16, no. 1, pp. 669-680, 2019.

[7] G. N. Schroeder, C. Steinmetz, C. E. Pereira, and D. B. Espindola, "Digital twin data modeling with AutomationML and a communication methodology for data exchange," IFAC-PapersOnLine, vol. 49, no. 30, pp. 12-17, 2016.

[8] W. Kritzinger, M. Karner, G. Traar, J. Henjes, and W. Sihn, "Digital Twin in manufacturing: a categorical literature review and classification," IFAC-PapersOnLine, vol. 51, no. 11, pp. 1016-1022, 2018.

[9] R. Söderberg, K. Wärmefjord, J. S. Carlson, and L. Lindkvist, "Toward a Digital Twin for real-time geometry assurance in individualized production," CIRP Annals, vol. 66, no. 1, pp. 137-140, 2017.

[10] A. K. Ghosh, A. M. M. S. Ullah, and A. Kubo, "Hidden Markov model-based digital twin construction for futuristic manufacturing systems," Artificial Intelligence for Engineering Design, Analysis and Manufacturing, vol. 33, no. 3, pp. 317331, 2019.

[11] G. Bachelor, E. Brusa, D. Ferretto, and A. Mitschke, "Modelbased design of complex aeronautical systems through digital twin and thread concepts," IEEE Systems Journal, vol. 14, no. 2, pp. 1568-1579, 2019.

[12] A. Castellani, S. Schmitt, and S. Squartini, "Real-world anomaly detection by using digital twin systems and weakly supervised learning," IEEE Transactions on Industrial Informatics, vol. 17, no. 7, pp. 4733-4742, 2020.

[13] R. Dong, C. She, W. Hardjawana, Y. Li, and B. Vucetic, "Deep learning for hybrid $5 \mathrm{G}$ services in mobile edge computing systems: learn from a digital twin," IEEE Transactions on Wireless Communications, vol. 18, no. 10, pp. 4692-4707, 2019.

[14] F. Tao, F. Sui, A. Liu et al., "Digital twin-driven product design framework," International Journal of Production Research, vol. 57, no. 12, pp. 3935-3953, 2019.

[15] J. Lee, M. Azamfar, J. Singh, and S. Siahpour, "Integration of digital twin and deep learning in cyber-physical systems: towards smart manufacturing," IET Collaborative Intelligent Manufacturing, vol. 2, no. 1, pp. 34-36, 2020.

[16] J. Bao, D. Guo, J. Li, and J. Zhang, "The modelling and operations for the digital twin in the context of manufacturing," Enterprise Information Systems, vol. 13, no. 4, pp. 534-556, 2019.
[17] A. Bilberg and A. A. Malik, "Digital twin driven human-robot collaborative assembly," CIRP Annals, vol. 68, no. 1, pp. 499-502, 2019.

[18] D. Guerra-Zubiaga, V. Kuts, K. Mahmood, A. Bondar, N. Nasajpour-Esfahani, and T. Otto, "An approach to develop a digital twin for industry 4.0 systems: manufacturing automation case studies," International Journal of Computer Integrated Manufacturing, vol. 34, no. 9, pp. 933-949, 2021.

[19] K. Y. H. Lim, P. Zheng, and C.-H. Chen, "A state-of-the-art survey of Digital Twin: techniques, engineering product lifecycle management and business innovation perspectives," Journal of Intelligent Manufacturing, vol. 31, no. 6, pp. 1313-1337, 2020. 